Draft Version OCtOBER 31, 2018

Preprint typeset using $\mathrm{HAT}_{\mathrm{E} X}$ style emulateapj v. 12/14/05

\title{
SEARCH FOR SUPERNOVA NEUTRINO BURSTS AT SUPER-KAMIOKANDE
}

\section{The Super-Kamiokande Collaboration}

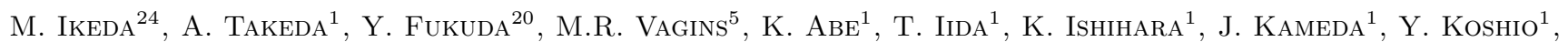
A. Minamino ${ }^{1}$, C. Mitsuda ${ }^{1}$, M. Miura ${ }^{1}$, S. Moriyama ${ }^{1}$, M. Nakahata ${ }^{1}$, Y. Obayashi ${ }^{1}$, H. OGawa ${ }^{1}$, H. Sekiya ${ }^{1}$, M. Shiozawa ${ }^{1}$, Y. Suzuki ${ }^{1}$, Y. Takeuchi ${ }^{1}$, K. Ueshima $^{1}$, H. Watanabe ${ }^{1}$, S. Yamada $^{1}$, I. Higuchi $^{2}$, C. Ishihara $^{2}$, M. Ishitsuka ${ }^{2}$, T. Kajita ${ }^{2}$, K. Kaneyuki ${ }^{2}$, G. Mitsuka ${ }^{2}$, S. Nakayama ${ }^{2}$, H. Nishino ${ }^{2}$, K. Okumura ${ }^{2}$, C. Saji ${ }^{2}$,

Y. Takenaga ${ }^{2}$, S. Clark ${ }^{3}$, S. Desai ${ }^{3, \dagger}$, F. Dufour ${ }^{3}$, E. Kearns ${ }^{3}$, S. Likhoded ${ }^{3}$, M. Litos ${ }^{3}$, J.L. RaAF ${ }^{3}$, J.L. Stone ${ }^{3}$, L.R. Sulak ${ }^{3}$, W. WAnG ${ }^{3}$, M. Goldhaber ${ }^{4}$, D. Casper ${ }^{5}$, J.P. Cravens ${ }^{5}$, J. Dunmore ${ }^{5}$, W.R. Kropp ${ }^{5}$, D.W. Wiu ${ }^{5}$,

S. Mine ${ }^{5}$, C. Regis ${ }^{5}$, M.B. SmY ${ }^{5}$, H.W. Sobel ${ }^{5}$, K.S. Ganezer ${ }^{6}$, J. Hill ${ }^{6}$, W.E. Keig ${ }^{6}$, J.S. JAnG ${ }^{7}$, J.Y. Kim ${ }^{7}$,

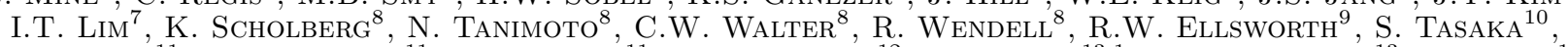

G. Guillian ${ }^{11}$, J.G. Learned ${ }^{11}$, S. Matsuno ${ }^{11}$, M.D. Messier ${ }^{12}$, Y. Hayato ${ }^{13,1}$, A. K. IChikawa ${ }^{13}$, T. Ishida ${ }^{13}$, T. Ishit ${ }^{13}$, T. Iwashita ${ }^{13}$, T. Kobayashi ${ }^{13}$, T. NAkAdaira ${ }^{13}$, K. NAKAmura ${ }^{13}$, K. Nitta ${ }^{13}$, Y. OYama ${ }^{13}$, Y. Totsuka ${ }^{13, \ddagger}$, A.T. Suzuki ${ }^{14}$, M. Hasegawa ${ }^{15}$, K. Hiraide ${ }^{15}$, I. Kato ${ }^{15, \S}$, H. Maesaka $^{15}$, T. Nakaya ${ }^{15}$, K. Nishikawa ${ }^{15}$, T. Sasaki ${ }^{15}$, H. Sato ${ }^{15}$, S. Yamamoto ${ }^{15}$, M. Yokoyama ${ }^{15}$, T.J. Haines ${ }^{16,5}$, S. DaZeley ${ }^{17}$, S. Hatakeyama ${ }^{17}$, R. Svoboda ${ }^{17}$, G.W. Sullivan ${ }^{18}$, D. Turcan ${ }^{18}$, A. Habig ${ }^{19}$, T. Tato ${ }^{20}$, Y. Itow ${ }^{21}$, T. Koike $^{21}$,

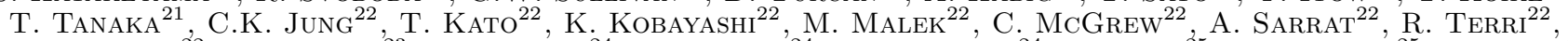
C. Yanagisawa ${ }^{22}$, N. Tamura ${ }^{23}$, Y. Idehara ${ }^{24}$, M. SAKudA ${ }^{24}$, M. Sugihara ${ }^{24}$, Y. Kuno ${ }^{25}$, M. YoshidA ${ }^{25}$, S.B. Kim ${ }^{26}$,

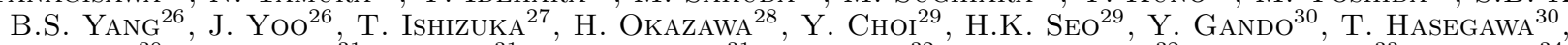

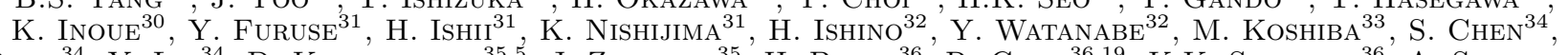
Z. DenG ${ }^{34}$, Y. Liu ${ }^{34}$, D. Kielczewska ${ }^{35,5}$, J. Zalipska ${ }^{35}$, H. Berns ${ }^{36}$, R. Gran ${ }^{36,19}$, K.K. Shiraishi ${ }^{36}$, A. Stachyra ${ }^{36}$, E. Thrane ${ }^{36}$, K. WAshburn ${ }^{36}$, R.J. Wilkes ${ }^{36}$

1 Kamioka Observatory, Institute for Cosmic Ray Research, University of Tokyo, Kamioka, Gifu, 506-1205, Japan

2 Research Center for Cosmic Neutrinos, Institute for Cosmic Ray Research, University of Tokyo, Kashiwa, Chiba 277-8582, Japan 3 Department of Physics, Boston University, Boston, MA 02215, USA

4 Physics Department, Brookhaven National Laboratory, Upton, NY 11973, USA

5 Department of Physics and Astronomy, University of California, Irvine, Irvine, CA 92697-4575, USA

${ }^{6}$ Department of Physics, California State University, Dominguez Hills, Carson, CA 90747, USA

7 Department of Physics, Chonnam National University, Kwangju 500-757, Korea

8 Department of Physics, Duke University, Durham, NC 27708, USA

9 Department of Physics, George Mason University, Fairfax, VA 22030, USA

10 Department of Physics, Gifu University, Gifu, Gifu 501-1193, Japan

11 Department of Physics and Astronomy, University of Hawaii, Honolulu, HI 96822, USA

12 Department of Physics, Indiana University, Bloomington, IN 47405-7105, USA

13 High Energy Accelerator Research Organization (KEK), Tsukuba, Ibaraki 305-0801, Japan

14 Department of Physics, Kobe University, Kobe, Hyogo 657-8501, Japan

15 Department of Physics, Kyoto University, Kyoto 606-8502, Japan

16 Physics Division, P-23, Los Alamos National Laboratory, Los Alamos, NM 87544, USA

17 Department of Physics and Astronomy, Louisiana State University, Baton Rouge, LA 70803, USA

18 Department of Physics, University of Maryland, College Park, MD 20742, USA

19 Department of Physics, University of Minnesota, Duluth, MN 55812-2496, USA

20 Department of Physics, Miyagi University of Education, Sendai, Miyagi 980-0845, Japan

21 Solar Terrestrial Environment Laboratory, Nagoya University, Nagoya, Aichi 464-8602, Japan

22 Department of Physics and Astronomy, State University of New York, Stony Brook, NY 11794-3800, USA

23 Department of Physics, Niigata University, Niigata, Niigata 950-2181, Japan

24 Department of Physics, Okayama University, Okayama, Okayama 700-8530, Japan

25 Department of Physics, Osaka University, Toyonaka, Osaka 560-0043, Japan

26 Department of Physics, Seoul National University, Seoul 151-742, Korea

27 Department of Systems Engineering, Shizuoka University, Hamamatsu, Shizuoka 432-8561, Japan

28 Department of Informatics in Social Welfare, Shizuoka University of Welfare, Yaizu, Shizuoka, 425-8611, Japan

29 Department of Physics, Sungkyunkwan University, Suwon 440-746, Korea

30 Research Center for Neutrino Science, Tohoku University, Sendai, Miyagi 980-8578, Japan

31 Department of Physics, Tokai University, Hiratsuka, Kanagawa 259-1292, Japan

32 Department of Physics, Tokyo Institute for Technology, Meguro, Tokyo 152-8551, Japan

33 The University of Tokyo, Tokyo 113-0033, Japan

34 Department of Engineering Physics, Tsinghua University, Beijing, 100084, China

35 Institute of Experimental Physics, Warsaw University, 00-681 Warsaw, Poland

36 Department of Physics, University of Washington, Seattle, WA 98195-1560, USA

Draft version October 31, 2018

\section{ABSTRACT}

The result of a search for neutrino bursts from supernova explosions using the Super-Kamiokande detector is reported. Super-Kamiokande is sensitive to core-collapse supernova explosions via observation of their neutrino emissions. The expected number of events comprising such a burst is $\sim 10^{4}$ and the average energy of the neutrinos is in few tens of $\mathrm{MeV}$ range in the case of a core-collapse supernova explosion at the typical distance in our galaxy (10 kiloparsecs); this large signal means that the detection efficiency anywhere within our galaxy and well past the Magellanic Clouds is $100 \%$. We examined a data set which was taken from May, 1996 to July, 2001 and from December, 2002 to October, 2005 corresponding to 2589.2 live days. However, there is no evidence of such a super- 
nova explosion during the data-taking period. The 90\% C.L. upper limit on the rate of core-collapse supernova explosions out to distances of 100 kiloparsecs is found to be $0.32 \mathrm{SN} \cdot$ year $^{-1}$.

Subject headings: galaxies: individual (our Galaxy,LMC,SMC) - neutrinos - supernovae: general

\section{INTRODUCTION}

On the 23rd of February, 1987, the Kamiokande II, IMB, and Baksan experiments observed the neutrino burst from SN1987A, which was located in the Large Magellanic Cloud (Hirata et al. 1987; Bionta et al. 1987; Alekseev et al. 1987) ${ }^{2}$. This was the first detection of a supernova's neutrino burst, and it introduced a new method of investigation: neutrino astronomy.

Super-Kamiokande (Super-K, SK) is an imaging water Cherenkov detector containing 50,000 tons of pure water; it is the successor to the Kamiokande detector. Super$\mathrm{K}$ is located 1000 meters underground (2,700 meters of water equivalent) in the Kamioka zinc mine in the Gifu prefecture of Japan, at $36.4^{\circ} \mathrm{N}, 137.3^{\circ} \mathrm{E}$ and $25.8^{\circ} \mathrm{N}$ geomagnetic latitude. The detector consists of a main inner detector and an outer veto detector. Both detectors are contained within a cylindrical stainless steel tank $39.3 \mathrm{~m}$ in diameter $\times 41.4 \mathrm{~m}$ in height. The usual fiducial mass for neutrino measurements is 22.5 ktons with boundaries $2.0 \mathrm{~m}$ from the inner surface. The outer detector is also a water Cherenkov detector of 13,000 metric tons total mass. It surrounds the inner detector as a $4 \pi$ solid-angle anti-detector to detect any signals coming from outside of the detector and to shield against external gamma-rays and neutrons.

The data set for the analyses was taken during two periods. The first period started on the 1st of April, 1996, and terminated on the 15th of July, 2001. A total of 11,146 photo multipliers (PMT's) with 20-inch diameter photocathodes provided active light collection over $40 \%$ of the entire surface of the inner detector. This phase of the project is now referred to as Super-Kamiokande-I (SK-I). The second phase, Super-Kamiokande-II (SK-II), started on the 10th of December, 2002, and terminated on the 6th of October, 2005. A total of 5,182 20-inch PMT's, each protected by acrylic and fiber-reinforced plastic (FRP) cases, were mounted on the inner detector, providing $19 \%$ photocathode coverage during this period. In the outer detector, a total of 1,885 8-inch PMT's were installed during both periods. Due to rapid variations in the water transparency, the data before the 31st of May, 1996 in SK-I, and the data before the 23rd of December, 2002 in SK-II have not been used for analysis because of uncertainties in the energy calibration. Having a welldefined energy response is necessary when searching for low multiplicity event clusters, as will be described in the later sections of this paper.

Theoretical calculations predict the characteristics of neutrinos expected from a supernova. A typical corecollapse supernova explosion emits all types of neutrinos and has a total energy output of $\sim 3 \times 10^{53}$ ergs which would generate about 10,000 SK events $(9,000$ events

\footnotetext{
$\dagger$ Present address: Center for Gravitational Wave Physics, Pennsylvania State University, University Park, PA 16802, USA

2 The LSD detector observed a cluster of 5 events about 5 hours earlier, but the correlation between this signal and SN1987A is favored only by non-standard double-bang scenarios of stellar collapse (Dadykin et al. 1987).
}

without neutrino oscillation) in the case of a supernova at a distance of $10 \mathrm{kpc}$ from the earth. Neutrino oscillations enhance the overall flux we observe by $10 \%$ as higher-temperature $\nu_{\mu}\left(\bar{\nu}_{\mu}\right)$ and $\nu_{\tau}\left(\bar{\nu}_{\tau}\right)$ get fully mixed into the observable $\nu_{e}\left(\bar{\nu}_{e}\right)$ signal.

These supernova events will be detected via the following interactions in SK, where the numbers in parentheses show the fractions of the total number of events with/without neutrino oscillations,

$$
\begin{aligned}
& \bar{\nu}_{e}+p \rightarrow n+e^{+} \quad(88 \% / 89 \%), \\
& \nu_{e}+e^{-} \rightarrow \nu_{e}+e^{-} \quad(1.5 \% / 1.5 \%), \\
& \bar{\nu}_{e}+e^{-} \rightarrow \bar{\nu}_{e}+e^{-} \quad(<1 \% /<1 \%), \\
& \nu_{x}+e^{-} \rightarrow \nu_{x}+e^{-} \quad(1 \% / 1 \%), \\
& \nu_{e}+{ }^{16} \mathrm{O} \rightarrow e^{-}+{ }^{16} \mathrm{~F} \quad(2.5 \% /<1 \%) \text {, } \\
& \bar{\nu}_{e}+{ }^{16} O \rightarrow e^{+}+{ }^{16} N \quad(1.5 \% / 1 \%) \text {, and } \\
& \nu_{x}+{ }^{16} O \rightarrow \nu_{x}+O^{*} / N^{*}+\gamma(5 \% / 6 \%),
\end{aligned}
$$

and $\nu_{x}$ means the total interactions of $\nu_{\mu}, \nu_{\tau}$, and their anti-neutrinos. To obtain these fractions (Takahashi et al. 2001), a supernova neutrino burst using the Livermore model (Totani et al. 1998) is assumed. To account for neutrino oscillations in Ref. (Takahashi et al. 2001), $\theta_{12}$ and $\Delta m_{12}^{2}$ are set to be in the favored solar neutrino LMA region, and $\theta_{23}$ and $\Delta m_{23}^{3}$ are determined from the best oscillation fit for the atmospheric neutrinos. For the fraction of the neutral current interactions with oxygen (Eq. 7), we look to Ref.(Langanke et al. 1996) and obtain our number by scaling their result to the numbers in Ref. (Takahashi et al. 2001). At present, based on both data and models, many of these numbers are uncertain up to a factor of two, especially the numbers of interactions with oxygen. In particular, Eqs.5, 6, and 7 are very dependent on the neutrino temperatures and details of mixing as discussed in the cited literature.

Averaged energies of neutrinos from a standard delayed explosion supernova model are expected to be between $11 \sim 26 \mathrm{MeV}$ (Totani et al. 1998; Thompson et al. 2003; Sumiyoshi et al. 2005) For example, the calculation performed by the Livermore group shows $\left\langle E_{\nu_{e}}\right\rangle \sim 11 \mathrm{MeV},\left\langle E_{\bar{\nu}_{e}}\right\rangle \sim 16 \mathrm{MeV}$ and $\left\langle E_{\nu_{\mu / \tau}}\right\rangle \sim 25$ $\mathrm{MeV}$ (Totani et al. 1998). The time profile of each type of neutrino has a unique shape. During the initial $\sim 10$ milliseconds, electron neutrinos from the neutronization are released with a total energy on the order of $10^{51}$ ergs. After the neutronization, all flavors of neutrinos are produced by electron-positron annihilation, and released with a total energy on the order of $10^{53} \mathrm{ergs}$ with a time scale of several tens of seconds.

It still is not clear at present, however, whether or not the delayed explosion scenario is the correct picture, in which a stalled shock wave will be reheated by energy deposition from neutrinos. We expect that the observation of a galactic supernova by the SK detector with high statistics will solve this long-standing question and, furthermore, shed light on other important questions such as neutrino oscillations and neutrino mass (Raffelt 2002). 
In this paper, we have searched for supernova neutrino bursts using the SK detector, and we present an upper limit on the supernova explosion rate within $100 \mathrm{kpc}$ of the earth (this volume contains our Galaxy, the $\mathrm{LMC}$, and the SMC). We also report the first result of search for neutronization bursts. Previously, AMANDA, Baksan, IMB, and MACRO have reported upper limits on the supernova explosion rate just within our Galaxy (Ahrens et al. 2002; Alekseev et al. 2002; Dye et al. 1989; Ambrosio et al. 2004), while LVD and Kamiokande have reported preliminary results (LVD Coll 2003; Suzuki 1993) of the same nature.

\section{DATA ANALYSIS}

\subsection{Data set}

From the 31st of May, 1996, to the 15th of July, 2001, and from the 24th of December, 2002, to the 5th of October, 2005, the livetimes of our detector for supernova searches were 1703.9 days for SK-I, and 885.3 days for SK-II. Livetime efficiency as a function of date is shown in Figure 1 - over the course of the entire data-taking period the average efficiency was about $89 \%$. The primary cause of lost supernova livetime was scheduled calibration work. The large losses of livetime seen in Figure 1 around July 1997, December 1998, June 1999, May 2001, March 2003, and September 2005 were due to LINAC energy calibration runs (Nakahata et al. 1999), though the biggest dip in 2003 was due to electronics problems which have since been corrected.

Vertex and energy reconstruction techniques are the same as those used in our solar neutrino analysis (Hosaka et al. 2006). Fiducial volume for the supernova search is also $22.5 \mathrm{ktons}$, though the energy thresholds are $6.5 \mathrm{MeV}$ (SK-I) and 7.0 MeV (SK-II) to avoid the higher background rates associated with the lower thresholds used in the solar neutrino analysis. Data reduction steps are basically the same. The first reduction includes removing events due to electronics noise, events with a poor reconstruction of the vertex position, and events with a vertex outside of the fiducial volume. A spallation cut removes events which are produced by energetic muons by using a likelihood method in which both the time difference $\Delta T$ and distance $\Delta L$ between the parent muon and subsequent events are considered (Hosaka et al. 2006). Mis-reconstructed events are also removed. After these noise reductions, the remaining event rates are 180 events/day for SK-I, and 164 events/day for SK-II.

\subsection{Analysis method}

In this section, we introduce our general method of supernova burst search. The procedure of data analysis is as follows:

1. Scan event times in the data set using a sliding time-window. We define a "cluster" if the number of events (the "multiplicity") within a time-window is greater or equal to a certain threshold.

2. Check each cluster found in the first step to determine if it is a real signal from a supernova or the result of background events.

A background cluster consists of either time-correlated non-supernova events or a chance coincidence of uncor-

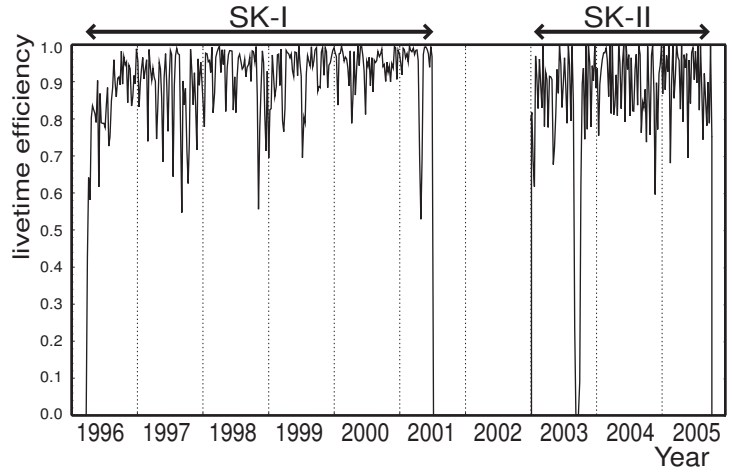

FIG. 1.- SK livetime efficiency as a function of time. Most of the big dips in efficiency were due to planned calibration runs such as LINAC energy calibration, though the biggest dip in 2003 was due to electronics problems.

related low energy events. There are two dominant background sources which produce time-correlated events. One such source is flasher PMT's which act as sources of light, and the other is spallation product, radioactive isotopes made via interactions between energetic cosmic ray muons and oxygen nuclei. In both cases, the reconstructed vertices of the resulting events are spatially concentrated. On the other hand, events made by actual supernova neutrinos should be generated uniformly in the detector volume. Therefore, to distinguish real signals from background clusters, clusters are checked by studying the correlation between the multiplicity and the events' spatial distribution $\left(R_{\text {mean }}\right)$. $R_{\text {mean }}$ is defined by the averaged spatial distance between each event as follows:

$$
R_{\text {mean }}=\frac{\sum_{i=1}^{M-1} \sum_{j=i+1}^{M}\left|\overrightarrow{r_{i}}-\overrightarrow{r_{j}}\right|}{{ }_{M} C_{2}} .
$$

where $M$ is the multiplicity, $\left|\overrightarrow{r_{i}}-\overrightarrow{r_{j}}\right|$ is the distance between events $i$ and $j$, and ${ }_{M} C_{2}$ is the number of unique combinations. In the case of a supernova burst, $R_{\text {mean }}$ should have a larger value than that resulting from spatially clustered events such as spallation events or flasher events.

Figure 2 shows $R_{\text {mean }}$ distributions of simulated supernova events for multiplicity $2,3,4$, and 8 , which are the multiplicity thresholds of various burst searches discussed in later sections of this paper. As shown in this figure, if the events in a cluster occur uniformly in the detector, as in supernova, then the expected value of $R_{\text {mean }}$ tends to be large, around $1,800 \mathrm{~cm}$. The threshold of $R_{\text {mean }}$ for a cluster with multiplicity equal to 2 is set to $750 \mathrm{~cm}$, and for clusters with multiplicity greater than 2 the threshold is set to $1,000 \mathrm{~cm}$. The efficiencies for supernova events using these criteria are $94 \%, 96 \%, 99 \%$, and $100 \%$, respectively.

In the following sections, we obtain results from three kinds of burst searches by changing the width of the timewindow and the multiplicity threshold and setting additional criteria for respective searches. The three burst searches are 1) distant supernova search, 2) supernova burst search with low energy threshold, and 3) neutronization burst search.

\subsection{Distant supernova search}




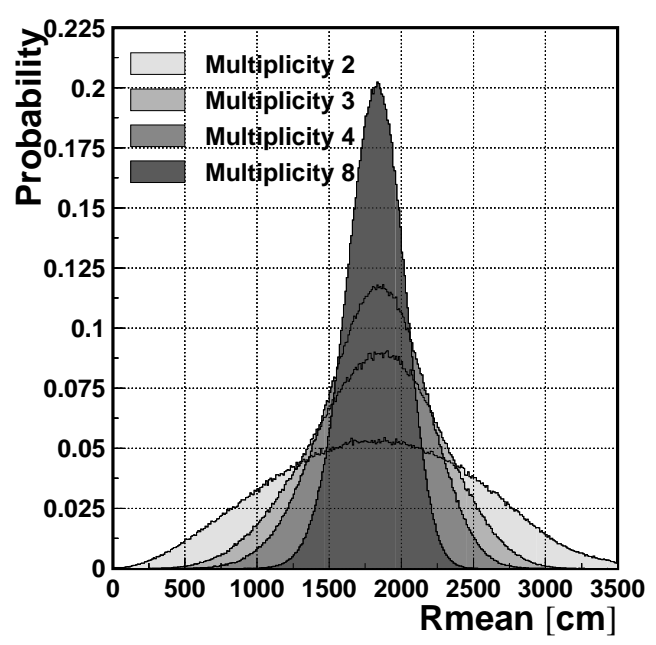

FIG. 2.- Expected $R_{\text {mean }}$ distributions of supernova events obtained by Monte Carlo simulation for multiplicity equal to 2, 3 , 4 , and 8 .

In recent years, a lot of effort has been put into optical supernova searches, with the result that the number of detected supernovae has increased substantially. The optical detection rate of supernovae suggests that theoretical calculations of supernova rates might be significantly underestimated. More specifically, nine corecollapse supernovae have been found in nearby galaxies between 2002 and 2005; this is three times higher than one theoretical estimate (Ando et al. 2005).

This motivates us to search for neutrinos from supernovae in nearby galaxies. Since the expected total number of events at SK from a supernova in the Andromeda galaxy $(\sim 700 \mathrm{kpc})$ is around two events, for this search we set our criteria as a long time-window and low multiplicity threshold such as $\geq 2$ events / 20 seconds. A lot of background clusters would be found in our usual solar neutrino data set (Hosaka et al. 2006) with this criterion. Therefore, we reduce those backgrounds by setting a higher energy threshold because the average energy of the emitted positron from the interaction (Eq.1) is higher than that of most low energy background events. To find the best energy threshold, we calculate the detection probability of a supernova and the number of chance coincidences of the low energy background events as functions of energy threshold value. Then we take the value of energy threshold which gives us the maximum value of $\frac{\text { Detection probability }}{\sqrt{\text { \#of chance coincidences }}}$ as an optimized energy threshold. The resulting value we set is $17 \mathrm{MeV}$, where the single event rate with this energy threshold is 0.762 event/day for SK-I, and 1.03 event/day for SK-II.

Figure 3 is a scatter plot of $R_{\text {mean }}$ versus the multiplicity for each cluster which satisfies the criteria with an energy threshold of $17 \mathrm{MeV}$. Three candidate clusters exist that have $R_{\text {mean }}$ more than $1000 \mathrm{~cm}$, but as shown in Table 1, the event times of those candidates actually coincide with times of mine blasting. Because of the physical vibration of PMT's due to blasting, huge electrical noise occurred, and those noises caused timeclustered a few hundreds events within a few seconds during or after blasting. It was also confirmed that these
TABLE 1

DETECTION TIME OF CANDIDATES IN SK-I

\begin{tabular}{cccc}
\hline \hline Candidate & Date and Time (JST) & Rmean & Multiplicity \\
\hline No.1 & Jul.13th 1999 19:00 & 1257.89 & 4 \\
No.2 & May.12th 2000 11:06 & 1928.76 & 35 \\
No.3 & Oct.12th 2000 19:03 & 1381.76 & 2 \\
\hline
\end{tabular}

a The blastings by Kamioka Mining and Smelting Company were scheduled at 11:00, 19:00, and 23:00, and shift takers reported that there were blastings at those times.

events including the candidate cluster events have a characteristic PMT hit pattern naerly in the same area of the inner detector, and hence they should be eliminated as potential supernova events.

The background clusters which have small $R_{\text {mean }}$ values in Fig. 3 were mainly found around times of calibration work, and more importantly the positions of these clusters were consistent with the positions of the calibration sources themselves. That these clusters made it into our supposedly all non-calibration data sample is therefore judged to be the result of occasional operator error in assigning the proper run type during transitions between normal and calibration data taking. (Note that such mis-labeled runs are excluded in our usual solar neutrino data analysis, but in order to maximize supernova livetime a wider variety of runs were included in this supernova analysis.) Therefore, no real supernova signal was observed during the data-taking periods in both SK-I and SK-II.

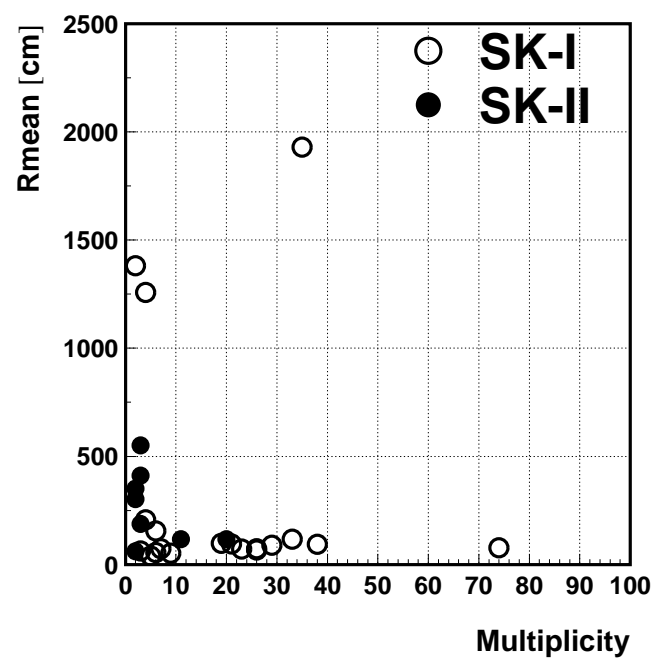

FIG. 3.- Correlation between the multiplicity and $R_{\text {mean }}$ for obtained clusters from data (open circle: SK-I, closed circle: SK-II ). There were 19 clusters observed in SK-I and 8 clusters in SK-II.

\subsection{Supernova burst search with low energy threshold}

In this section, we set various time-windows of 0.5 , 2 , and 10 seconds with a lower energy threshold of 6.5 $\mathrm{MeV}$ for SK-I and 7.0 MeV for SK-II to search for signals from a supernova in this lower energy region. In fact, the data of SN1987A imply $\langle E\rangle=7.5 \mathrm{MeV}$ at Kamiokande 
and $11.1 \mathrm{MeV}$ at IMB (Jegerlehner et al. 1996) while $\left\langle E_{\bar{\nu}_{e}}\right\rangle \sim 16 \mathrm{MeV}$ to take the Livermore group model as an example (Totani et al. 1998).

Since a lot of background events due to spallation or flasher PMT's still remain in the lower energy regions, we set criteria of higher multiplicity for each time-window as $\geq 3$ events / 0.5 seconds, $\geq 4$ events / 2.0 seconds, and $\geq$ 8 events/ 10 seconds. If a cluster satisfies the requirement $R_{\text {mean }}$, we closely check data around the cluster - for example all events within \pm 20 seconds around the cluster - so that we get information from as many supernova neutrinos as possible.

Figure 4 shows the correlation between $R_{\text {mean }}$ and multiplicity for obtained clusters from SK-I and SK-II. There were only three clusters which had large $R_{\text {mean }}$ values in SK-I. As mentioned in the previous section, however, all three candidate clusters consist of the same events as in the previous section which were found during periods of mine blasting.

The same criteria are applied to SK-II data, and the correlation of the multiplicity and $R_{\text {mean }}$ of the candidate clusters was studied. There was no cluster with $R_{\text {mean }} \geq$ $1000 \mathrm{~cm}$, but two candidate clusters had $R_{\text {mean }}$ just below $1000 \mathrm{~cm}$. To make sure that those candidates were not real signals, cosmic ray muon events around the cluster were studied, and event displays of candidate events were checked. As a result, one of the candidates was found to be an accidental coincidence of a low energy event with spallation events. Although most spallation events have already been removed by the spallation cut, a small fraction of spallation products are still present at lower multiplicities. The other candidate cluster was comprised of false events caused by a flasher tube. As in the previous search, it was confirmed that the background clusters with small $R_{\text {mean }}$ were mostly due to mislabeled calibration work.

In conclusion, the remaining candidate clusters were all caused by mine blasting, and so there is no clear evidence for any supernova neutrino burst in the data obtained by SK-I as well as SK-II. Therefore, we will present an upper limit at the $90 \%$ confidence level for the rate of supernova explosions in Section 3.

\subsection{Neutronization burst search}

We conducted another burst search with shorter timewindows to investigate the short-lived neutronization burst of $\nu_{e}$ events from a supernova. Prior to the core explosion, many $\nu_{e}$ 's are emitted via the reaction $e^{-}+p \rightarrow \nu_{e}+n$ as the shock wave propagates into the exploding star's outer core. The shock wave dissociates nuclei into free nucleons on which the cross section of electron capture is larger than that on nuclei; the resulting burst of $\nu_{e}$ 's thus forms the so-called neutronization burst.

The duration of the neutronization burst is on the time scale of the shock wave propagation, which is less than 10 milliseconds. Hence, if such a short time window is set, the $\nu_{e}$ 's can be dominant, and the expected number of neutronization burst neutrinos which will be observed at SK is between one and six (depending on neutrino oscillation models) in the case of a supernova at a distance of $10 \mathrm{kpc}$ from the earth (Takahashi et al. 2001). It should be mentioned that even if no supernova explosion is observed in the galaxy during that period, it

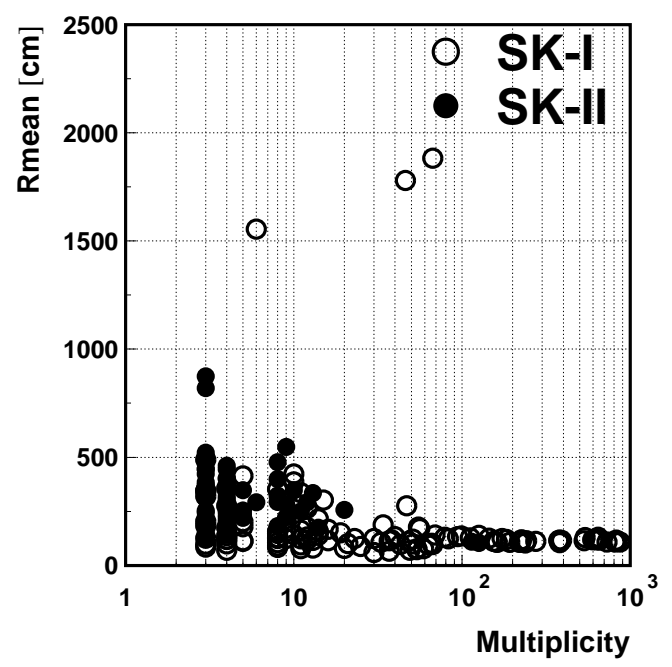

Fig. 4.- Correlation between the multiplicity and $R_{\text {mean }}$ for obtained clusters from data (open circle: SK-I, closed circle: SKII). There are 121 and 53 clusters observed in SK-I and SK-II respectively. If a cluster satisfies more than one criterion, the circle for the cluster represents the largest multiplicity, and $R_{\text {mean }}$ for the cluster is calculated from the multiplicity of events.

still might be possible to observe only the neutronization burst. For example, if a black hole forms shortly after the neutronization stage, then the main burst of supernova neutrinos might not be able to escape from the black hole (Sumiyoshi et al. 2006).

Based on the above theoretical expectations, we set time-windows of 1,10 , and 100 milliseconds. The multiplicity threshold is two events for each time window; $\geq$ 2 events / $1 \mathrm{msec}, \geq 2$ events / $10 \mathrm{msec}$, or $\geq 2$ events / 100 msec. Because recoil electrons will have lower energies from neutrino-electron scattering (Eq.2) which is the dominant interaction in this case, we use the same sample as the SK-I solar neutrino analysis (Hosaka et al. 2006), whose energy threshold is $5 \mathrm{MeV}$ and livetime is 1496 days, and we use the same sample as in previous sections for SK-II.

Since the threshold of multiplicity for a candidate cluster is two events, a lot of backgrounds exist even after the $R_{\text {mean }}$ cut. Therefore, we need another variable to reduce background. In this case, the directional information of observed events is a strong tool because the recoil electrons have almost the same direction as the incident neutrinos, which means that events from a real neutronization burst should have roughly the same reconstructed direction. To check the isotropy of events in a candidate cluster, a new variable is defined as follows:

$$
\text { Sumdir }=\frac{\left|\sum_{i=1}^{M} d \overrightarrow{i r}_{i}\right|}{M} \text {. }
$$

where $d \overrightarrow{i r}_{i}$ is a reconstructed direction vector of events in a cluster, and $M$ is the multiplicity of the cluster . By this definition, Sumdir will be close to 1 in the case of a real supernova cluster. Figure 5 shows the Sumdir distributions of supernova Monte Carlo and background Monte Carlo. We set the threshold of Sumdir for a supernova candidate cluster to 0.75 as this is the point at 
which the signal-to-noise ratio exceeds unity; the efficiency for real supernova events is estimated to be $84 \%$.

Table 2 shows the number of observed candidates during the periods of SK-I and SK-II after $R_{\text {mean }}$ and Sumdir cuts. There is good agreement between the number of observed clusters and the number of expected backgrounds which can be estimated by

$$
N_{b g}=\sum_{i=\text { start }}^{\text {stop }} \sum_{j=M_{\text {thr }}}^{\infty} R_{i} T_{i} \frac{e^{-R_{i} \Delta T}\left[R_{i} \Delta T\right]^{j-1}}{(j-1) !}
$$

where $M_{t h r}$ is threshold of multiplicity, $R_{i}$ and $T_{i}$ are single event rate and live time of each data taking period (maximum 24 hours), and $\Delta T$ is the time-window for each case. We can use a Poisson-based estimate for the background because, after the $R_{\text {mean }}$ cut, most of the background clusters are due to chance coincidences of low energy events such as solar neutrino events, flasher events, and spallation events in the data samples. Since there were no candidates with stricter criteria: $\geq 3$ events / 1 msec, $\geq 3$ events / 10 msec, or $\geq 3$ events / 100 msec, also in agreement with the expected background as shown in Table 2, we conclude that no signal from a real neutronization burst was observed during this period.

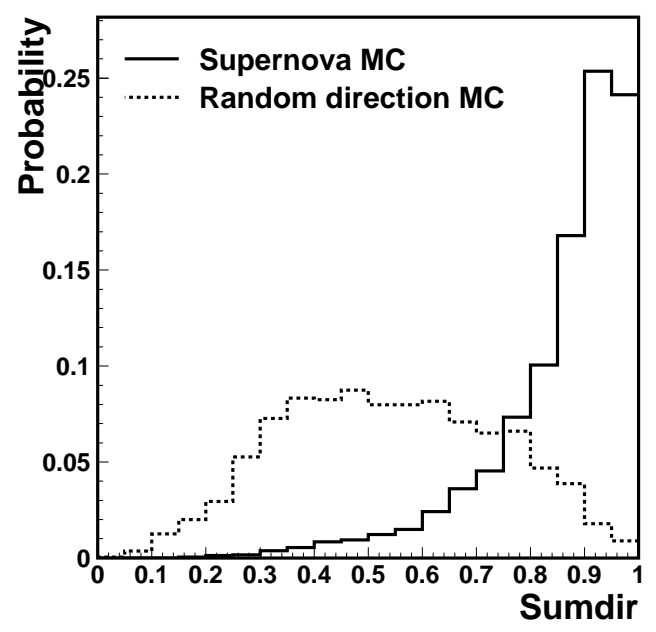

FIG. 5.- The distribution of Sumdir. The solid line shows the histogram of supernova Monte Carlo events where the incident electron neutrinos from a neutronization burst have a fixed direction. The dashed line shows the histogram of random direction events in the SK tank.

\section{DISCUSSION AND CONCLUSIONS}

\footnotetext{
3 Even during periods of calibration we found no clear galactic supernova neutrino bursts in the SK detector.
}

We have searched the SK-I and SK-II data for neutrino burst signals from supernova explosions. We conclude that no real signals of supernova bursts occurred during the data taking periods between late May 1996 and early October 2005, which corresponds to a total livetime of 2589.2 days $^{3}$. Super-K also performed an all sky search for transient astrophysical neutrinos in the GeV-TeV energy region and did not find anything (Abe et al. 2006), so we can rule out the detection of high energy neutrinos from any supernovae as well.

We can evaluate the performance of Super-K as a supernova watcher based on these results. We simulate neutrino events in the tank to estimate detection probability of a supernova as a function of distance from the earth. The incident neutrinos are assumed to be emitted by a supernova of the model used by the Livermore group (Totani et al. 1998). The detection probability of a supernova at a certain distance is determined as a probability in which one simulated neutrino burst satisfies each criterion given in previous sections after basic data reduction.

As shown in Figure 6, full detection probability is maintained out to around $100 \mathrm{kpc}$. Therefore, the upper limit at $90 \%$ C.L. for the supernova explosion rate out to $100 \mathrm{kpc}$ - within which our Galaxy, the LMC, and the SMC may be found - is determined to be 0.32 per year by combining the results from SK-I and SK-II. While the probability for the burst search with lower energy thresholds goes down rapidly to almost 0.0 at $700 \mathrm{kpc}-$ the distance to the Andromeda Galaxy - the probability of the distant supernova search is still 0.075 at this distance, which demonstrates the benefit of conducting a long time-window search in addition to the usual burst search.

We have also performed, for the first time, a systematic search for neutrinos from neutronization bursts. These could occur in isolation in the case of early black hole formation following a core collapse. However there was no such signal observed in the data set with a total livetime of $2,381.3$ days.

\section{ACKNOWLEDGMENTS}

The authors gratefully acknowledge the cooperation of the Kamioka Mining and Smelting Company. Super-K has been built and operated from funds provided by the Japanese Ministry of Education, Culture, Sports, Science and Technology, the U.S. Department of Energy, and the U.S. National Science Foundation. This work was partially supported by the Korean Research Foundation (BK21), the Korean Ministry of Science and Technology, and the National Science Foundation of China.

\section{REFERENCES}

Abe, K. et al, 2006, Astrophys. J. 652, 198.

Ahrens, J. et al, 2002, Astropart. Phys. 16, 345.

Alekseev, E. N. et al., 1987, Pisma Zh. Eksp. Teor. Fiz 45, 461. [JETP Lett. 45, 589 (1987)]

Alekseev, E. N. et al., 2002, Zh. Eksp. Teor. Fiz 95, 10. [2002, J.

Exp. Theor. Phys 95, 10

Ambrosio, M. et al., 2004, Eur. Phys. J. C 37, 265.

Ando, S. et al., 2005, Phys. Rev. Lett. 95, 171101.
Bionta, R. M. et al., IMB collaboration, 1987, Phys. Rev. Lett. 58, 1494.

Dadykin, V. L. et al., 1987, Pisma Zh. Eksp. Teor. Fiz 45, 464. [1987, JETP Lett. 45, 593 ]

Dye, S. T. et al., 1989, Phys. Rev. Lett. 62, 2069.

Hirata, K. S. et al., 1987, Phys. Rev. Lett. 58, 1490

Hosaka, J. et al., 2006, Phys. Rev. D 73, 112001

Jegerlehner, B. et al., 1996, Phys. Rev. D 54, 1194. 
TABLE 2

NUMBER OF CANDIDATES AND BACKGROUNDS IN NEUTRONIZATION BURST SEARCH

\begin{tabular}{ccccc}
\hline \hline & \multicolumn{2}{c}{ SK-I } & \multicolumn{2}{c}{ SK-II } \\
\cline { 2 - 5 } Criterion & Candidate & BG $^{\mathrm{a}}$ & Candidate & BG $^{\mathrm{a}}$ \\
\hline$\geq$ 2events $/ 1 \mathrm{msec}$ & 1 & 2.10 & 0 & 0.125 \\
$\geq$ 2events $10 \mathrm{msec}$ & 19 & 19.1 & 0 & 1.25 \\
$\geq 2$ events $/ 100 \mathrm{msec}$ & 194 & 191 & 10 & 12.5 \\
\hline$\geq$ 3events $/ 1 \mathrm{msec}$ & 0 & $9.90 \times 10^{-6}$ & 0 & $1.65 \times 10^{-7}$ \\
$\geq$ 3events/10msec & 0 & $9.78 \times 10^{-4}$ & 0 & $1.65 \times 10^{-5}$ \\
$\geq$ 3events $/ 100 \mathrm{msec}$ & 0 & $9.78 \times 10^{-2}$ & 0 & $1.65 \times 10^{-3}$ \\
\hline
\end{tabular}

aThe number of backgrounds was calculated from the chance coincidence rate of non-supernova signals such as solar neutrino events, flasher events, and spallation events in the data samples.

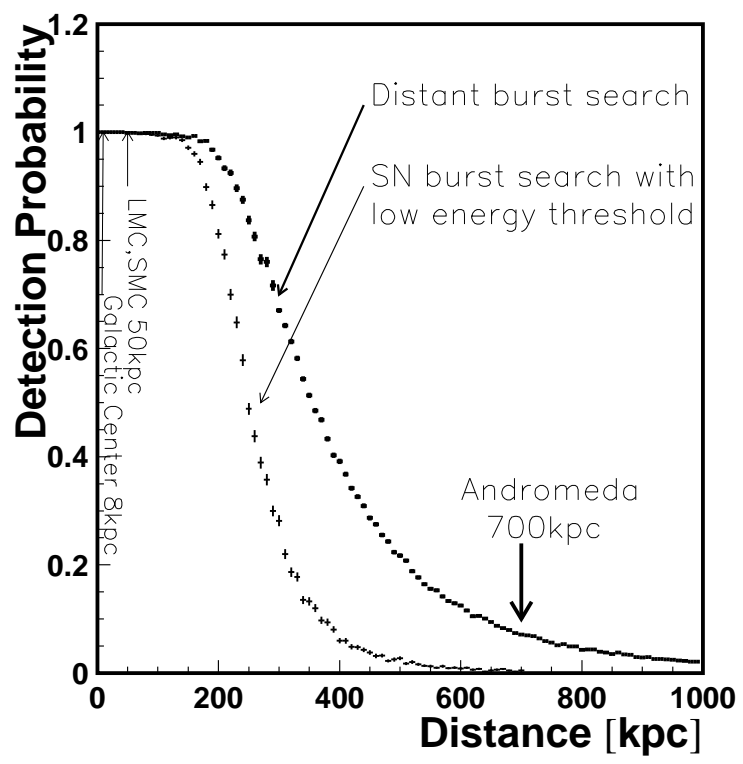

Langanke, K. et al., 1996, Phys. Rev. Lett. 76, 2629

LVD Coll, 2003, proc. 28th ICRC H.E 2.3.9, Tokyo.

Nakahata, M. et al., 1999, Nucl. Instrum. Methods Phys. Res. Sect. A 421, 113

Raffelt, G. G. Nucl. Phys. B (Pro. Suppl) 2002, 110, 254.

Sumiyoshi, K. et al., 2005, Astrophys. J. 629, 922.

Sumiyoshi, K. et al., 2006, Phys. Rev. Lett. 97, 91101.

Suzuki, Y. 1993, in Proc.of the International Symposium on Neutrino Astrophysics: Frontiers of Neutrino Astrophysics, edited by Y. Suzuki and K. Nakamura, (Universal Academy

Press Inc. Tokyo), number 5 in Frontier Science Series, p.61.

Thompson, T. A. et al., 2003, Astrophys. J. 592, 434.

Totani, T. et al., 1998, Phys. Rev. Lett. 80, 2039.

Takahashi, K. et al., 2001, Phys. Rev. D 64, 93004.

FIG. 6.- The probability of detecting supernovae assuming a specific supernova model at SK. Full (100\%) detection probability is retained out to around $100 \mathrm{kpc}$. 